\title{
Application of MALDI Tissue Imaging of Drugs and Metabolites: A New Frontier for Molecular Histology
}

\author{
Selina Rahman Shanta ${ }^{1}$, Youngjun $\mathrm{Kim}^{2}$, Young Hwan $\mathrm{Kim}^{3,4}$ and Kwang Pyo Kim ${ }^{1, *}$ \\ ${ }^{1}$ Department of Molecular Biotechnology, WCU Program, Konkuk University, Seoul 143-701, \\ ${ }^{2}$ Department of Applied Biochemistry, Konkuk University, Chungju 380-701, \\ ${ }^{3}$ Division of Mass Spectrometry Research, Korea Basic Science Institute, Ochang, 863-883, \\ ${ }^{4}$ Graduate School of Analytical Science and Technology, Chungnam National University, Daejeon 305-764, Republic of Korea
}

\begin{abstract}
Matrix assisted laser desorption ionization (MALDI) mass spectrometry is commonly used to analyze biological molecules such as proteins, peptides and lipids from cells or tissue. Recently MALDI Imaging mass spectrometry (IMS) has been widely applied for the identification of different drugs and their metabolites in tissue. This special feature has made MALDI-MS a common choice for investigation of the molecular histology of pathological samples as well as an important alternative to other conventional imaging methods. The basic advantages of MALDI-IMS are its simple technique, rapid acquisition, increased sensitivity and most prominently, its capacity for direct tissue analysis without prior sample preparation. Moreover, with ms/ms analysis, it is possible to acquire structural information of known or unknown analytes directly from tissue sections. In recent years, MALDI-IMS has made enormous advances in the pathological field. Indeed, it is now possible to identify various changes in biological components due to disease states directly on tissue as well as to analyze the effect of treated drugs. In this review, we focus on the advantages of MALDI tissue imaging over traditional methods and highlight some motivating findings that are significant in pathological studies.
\end{abstract}

Key Words: MALDI imaging, Metabolites, Lipids, Drugs

\section{INTRODUCTION}

The biological research field was reformed with the invention of Matrix assisted laser desorption ionization (MALDI) and electrospray ionization (ESI) mass spectrometry (MS) in 1980. This technique has since become a powerful tool for the analysis of large molecules such as proteins or peptides from tissue or cell lines (Caprioli et al., 1997; Chaurand, et al., 1999). However MALDI imaging (IMS), which was developed a decade later, has opened a new way of understanding. Previously, less focus was given to small metabolites such as exogenous drugs or endogenous metabolites such as lipids or amino acids due to the complex nature of these molecules and lack of tools for their analysis. However, since the invention of IMS, it has become possible to identify small metabolites directly from tissue sections, as well as to identify their spatial localization on tissues (Sugiura and Setou, 2010; Shanta et al., 2011).

Two main experimental protocols are used in the analysis of molecules directly from tissues by MALDI, profiling and im-

www.biomolther.org

Open Access DOI: 10.4062/biomolther.2011.19.2.149

pISSN: 1976-9148 elSSN: 2005-4483

Copyright $\odot 2011$ The Korean Society of Applied Pharmacology aging (Fig. 1). For MALDI an acidic aromatic matrix is applied to the surface of the target samples. The matrix is dissolved in organic solvent and $0.5-1 \mu \mathrm{l}$ matrix solution is dropped on the tissue section for profiling. MALDI profiling facilitates comparison of the distribution of target molecules between diseased and normal or control and treated cells over a short time. In MALDI-IMS experiments, the entire tissue is sprayed with matrix solution, dried and the resulting co-crystals of the matrix and analytes are then focused by short laser pulses, usually using an UV laser, which causes desorption and ionization of the analytes. The mass-to-charge ratio $(\mathrm{m} / \mathrm{z})$ is then measured using a time-of-flight mass analyzer (Chaurand et al., 2005; Walch et al., 2008; Schwamborn and Caprioli, 2010).

Although several methods have been developed to stain diseased tissue to distinguish normal and diseased regions, most of these require chemical or mass tagging for identification of specific molecules (Lee et al., 2009). The major advantages of MALDI-IMS over conventional imaging techniques are as follows: 1) MALDI-IMS does not require prior extraction of samples from the tissue; therefore, the localization informa-

Received Jan 23, 2011 Revised Apr 12, 2011 Accepted Apr 13, 2011

* Corresponding Author

E-mail: kpkim@konkuk.ac.kr

Tel: +82-2-458-7682, Fax: +82-2-450-3395 


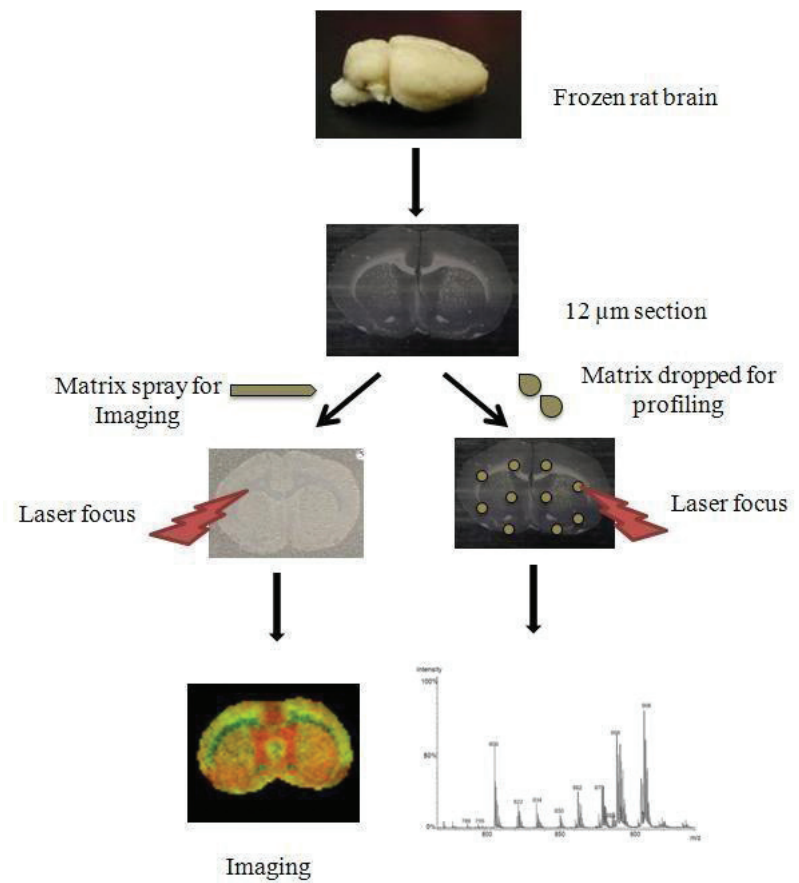

Fig. 1. General workflow with MALDI mass spectrometry. Frozen brain tissue is sectioned at a thickness of $12 \mu \mathrm{m}$ and mounted on an ITO slide. For imaging, the matrix solution is sprayed over the tissue section and in cases of profiling, matrix solution is dropped on the region of interest. After drying, when the matrix co-crystalized with analytes, a laser is focused on the crystal, which helps in desorption and ionization of analytes. After IMS experiments, whole tissue images for any analytes can be displayed.

tion of the molecules on the tissue is preserved; 2) No tagging steps are required for structural information regarding the target molecules; 3 ) It is possible to have immediate images of the target molecules on drugs and their metabolites from single tissue experiments, as well as to acquire the structural information of unknown metabolites by tandem mass spectrometry $(\mathrm{ms} / \mathrm{ms})$ directly from the tissue section.

Even though MALDI-IMS has several advantages over other techniques, still few technical and experimental developments are required. In case of analyzing samples by MALDIMS, some parameters are needed to consider, for example the selection of appropriate matrix, the solvent composition and the method of matrix application and deposition. These are very important factors because it can effect on data accuracy and reproducibility. The second reason is the separation of isobaric peak, which have the same $\mathrm{m} / \mathrm{z}$ value with different structure. However, with the application of previous separation techniques like HPLC or TLC and with the development of higher resolution MALDI-MS, it is possible to overcome this problem easily. Another most important technical problem arises with MALDI-IMS experiment is the imaging resolution. Currently $50 \mu \mathrm{m}$ resolution is widely used for tissue sample analysis which can be improved up to $1-5 \mu \mathrm{m}$ for sub cellular analysis.

In this review we focus on the recent development of imaging of small molecules and their metabolites, especially lipids, on tissue sections, by the use of MALDI-IMS.

\section{DIFFERENCE BETWEEN MALDI-IMS AND ESI MASS SPECTROMETRY}

Mass spectrometry is currently being revolutionized to analyze complex biological samples. The major difference between ESI and MALDI mass spectrometry is the sample ionization process.

In ESI mass spectrometry, biological molecules of interest are extracted from cells or tissues in organic solvent that is evaporated under a high electric field to facilitate ionization of the samples. In the case of tissue analysis due to this extraction process, location information regarding the identified components is lost. Conversely, in MALDI mass spectrometry, biological tissue samples can be analyzed either directly from tissue or after extraction of biomolecules of interest. To analyze any diseased or normal tissue samples directly without extraction, the specimens are sectioned and coated with matrix, after which they are subjected to the MALDI MS for analysis. As with MALDI mass spectrometry, it is possible to conduct profiling of biomolecules and whole tissue imaging, which provides information regarding the localization of specific molecules within the tissue section as well as their identification. At the same time, it is also possible to compare changes in the composition of any interested biomolecules due to the diseased condition on the tissue.

\section{IDENTIFICATION OF DRUGS ON TISSUES BY MALDI- IMS}

IMS of pharmaceutical compounds provides definitive answers regarding the localization of candidate drugs on a particular organ. Although traditional imaging techniques such as magnetic resonance imaging, autoradiography or fluorescence microscopy are widely used, these techniques have some limitations such as the need for the use of radioisotopic tags for identification of drugs and the relatively low resolution. Compare to traditional methods for example whole body autoradiography (WBA) technique, IMS gives similar drug distribution image without using radiolabeled compounds. At the same time, IMS can show the distribution of intact drug and their metabolites whereas WBA can't distinguished them. Additionally, IMS can picture drug distribution on tissue with much lower cost and with shortest time than using radiolabel isotope (Sugiura and Setou, 2010). Another widely used pharmaceutical method, PET, requires derivitization of drug compounds to incorporate radioisotope molecules for detection (Wiseman et al., 2008). In comparison, development of mass spectrometry provides us with a more convenient, accurate technique for detection and imaging of drugs and their metabolites. When different types of mass spectrometry such as GC-MS or LCMS techniques are used, drugs are analyzed by homogenizing the treated tissues followed by derivitization and extraction. These processes are laborious, time consuming and can influence the sample analysis. However, with MALDI IMS of tissue sections, no prior sample extraction or modification steps are needed; therefore, there are less analytical complications. In addition, MALDI IMS concurrently enables the exact location of the treated drug and their metabolites to be obtained.

Patients with mood disorder are generally treated with the prescription drug, olazapine (OLZ). Conventional methods have revealed that olazapine and its metabolites are distrib- 


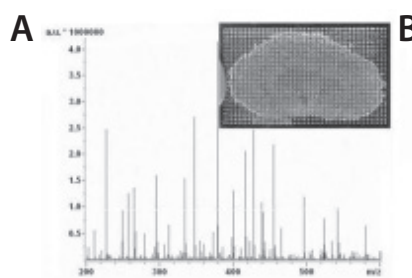

C
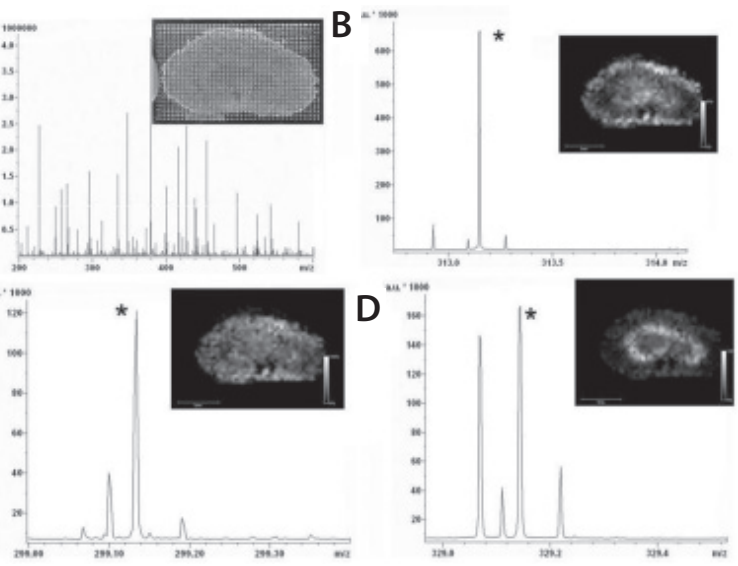

Fig. 2. Distribution of the drug olazapine and its metabolites on rat kidneys by MALDI-IMS (Cornett et al., 2008). (A) Total MS spectra from whole kidney tissue sections. The asterisks represent the protonated molecule of olanzapine and its ion distribution on the outer cortex region (B), ion distribution of the metabolite of olanzapine (C) and hydroxymethyl metabolites of olanzapine on the outer medull (D). (Reprinted with permission from Analytical Chemistry, 2008, 80, 5648, the American Chemical Society).

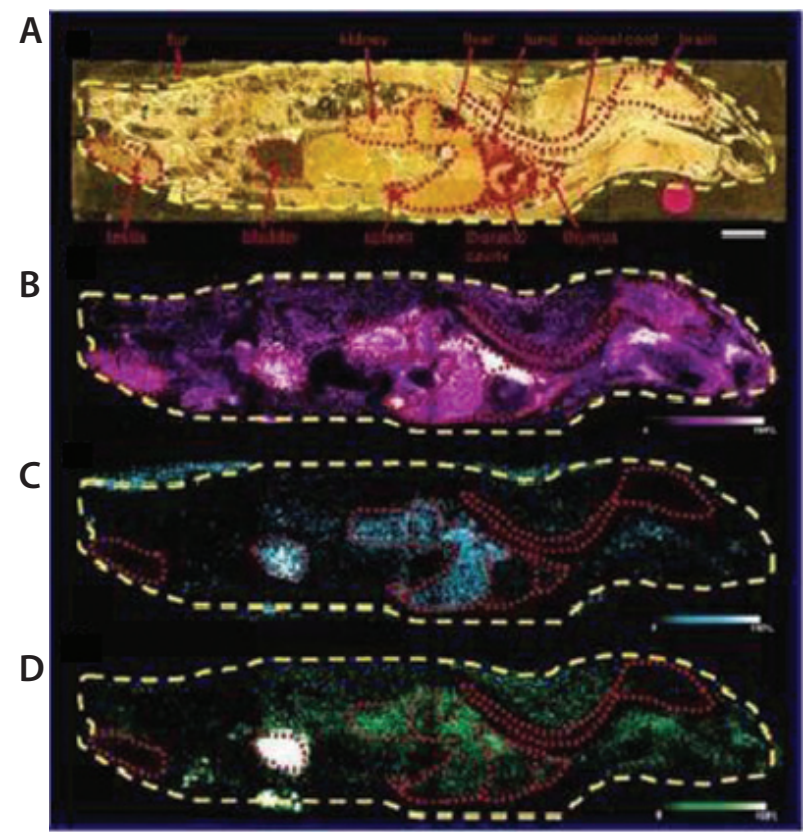

Fig. 3. Whole rat sagittal section imaging by MALDI mass spectrometry in a time dependent manner to show the candidate drug and its metabolite's targeted localization by Khatib- shahidi et al., 2006. (A) Optical image of $2 \mathrm{~h}$ drug treated sagittal whole body section. (B) Organ outlined and $\mathrm{ms} / \mathrm{ms}$ ion image of olanzapine at $\mathrm{m} / \mathrm{z} 256$. (C) $\mathrm{ms} / \mathrm{ms}$ ion image of $n$-desmethyl metabolite at $\mathrm{m} /$ z 256. (D) $\mathrm{ms} / \mathrm{ms}$ ion image of 2-hydroxymethylmetabolite at $\mathrm{m} / \mathrm{z}$ 272 (Khatib-Shahidi et al., 2006). (Reprinted with permission from Analytical Chemistry, 2006, 78, 6448, the American Chemical Society).

uted on different organs such as the brain, kidney, liver and spleen (Aravagiri et al., 1999). In a study conducted by Cornett et al., 344 ten week-old male Fischer rats were treated
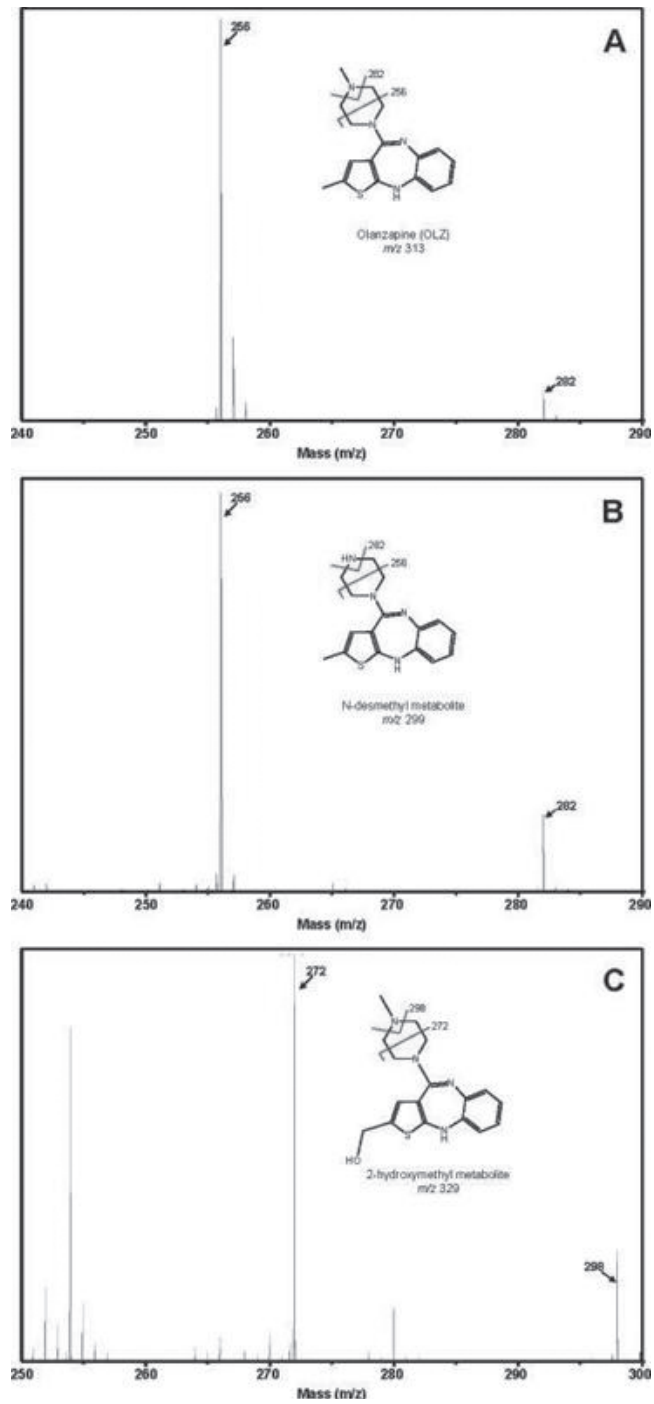

Fig. 4. $M S / M S$ spectra of $O L Z$ and its metabolites on tissue. (A) Olanzapine $\mathrm{m} / \mathrm{z} 313 \rightarrow 256$, (B) N-Desmethyl metabolite $\mathrm{m} / \mathrm{z} 299$ $\rightarrow 256$ and (C) 2-Hydroxymethyl metabolite $\mathrm{m} / \mathrm{z} 329 \rightarrow 272$ (Khatib-Shahidi et al., 2006). (Reprinted with permission from Analytical Chemistry, 2006, 78, 6448, the American Chemical Society).

with olazapine, sacrificed after $2 \mathrm{~h}$ of drug treatment and IMS experiments were then conducted on kidney tissues. Intact OLZ was found along the outer cortex; however, their metabolites were found to be abundant on the outer medulla (Fig. 2). This type of specific molecular location information cannot be obtained by any traditional methods (Cornett et al., 2008).

Khatib-shahidi et al. (2006) examined the molecular distribution of the same OLZ drug and their metabolites in the whole rat sagittal section in a time dependent manner by MALDI IMS. Using IMS experiments, the author demonstrated that the intact OLZ successfully reached the targeted organ (brain), although their metabolites were distributed all over the body as shown in Fig. 3. With whole body autoradiography, it is not possible to show this type of molecular specific localization of candidate drugs and their metabolites. Due to identify fragmentation pattern of OLZ and its metabolites from collision induced dissociation (CID) of parent molecules, on tissue drug 
spot was analyzed and the obtained spectra were shown in Fig. 4 (Khatib-Shahidi et al., 2006).

Wang et al. (2005) also showed the identification of small drug molecules by MALDI mass spectrometry. To establish MALDI as an effective technique compared to traditional techniques, they treated rat with intracranial chlorisondamine which is a nicotinic antagonist that causes neuronal and ganglionic blockage and intraperitoneal cocaine which is an alkaloid that stimulates the central nervous system injections. After treatment, the rat was sacrificed and rat brain was analyzed by MALDI-MS to identify cocaine and chlorisondamine on brain tissue. For identification of these drugs, the CHCA matrix was used for chlorisondamine and the DHB matrix was used for cocaine, respectively. The results suggested that the optimal matrix application improved the accuracy of drug identification on tissue. Initially, they used $1 \mathrm{nmol} / \mu \mathrm{l}$ chlorisondamine with CHCA matrix and found peaks at $\mathrm{m} / \mathrm{z} 356.2,358.2,360.2$ and 362.2 due to the four chlorine atoms present in chlorisondamine. Using an IMS experiment on brain tissue, they successfully identified all peaks except for one on the ventral tegmental area. In addition, they detected cocaine at $\mathrm{m} / \mathrm{z} 304.4$ and to confirm their structure by performing $\mathrm{ms} / \mathrm{ms}$ analysis of $\mathrm{m} / \mathrm{z}$ 304.4 on tissue and found identical fragments at $\mathrm{m} / \mathrm{z} 105.4$, 124.2, 166.1 and 182.1 (Wang et al., 2005).

\section{METABOLOMICS AND ITS PERSPECTIVES: LIPIDS}

The growing field known as metabolomics defines and detects small molecules known as metabolites that are produced by living cells under different physiological conditions of their life cycle. The term metabolomics has only existed for less than a decade, but has recently been the focus of a great deal of attention. The most effective and well known field is lipidomics, in which research is currently being conducted to detect and quantify the lipid composition of cells under various physiological conditions. Recently, the development of protocols based on mass spectrometry has provided an essential tool for lipid analysis. However, mass spectrometric methodologies for endogenous metabolites such as lipid identification are still undergoing development.

Lipids, especially phospholipids, are important constituents of cellular membrane. Among them, diacylglycerol and phosphotidylinosiols work as a secondary messengers in cell signaling processes (Kim et al., 2010), and lysophosphotidylcholines have been recognized as disease biomarkers for atherosclerosis and rheumatoid arthritis. In addition, because they play a major role as building block molecules in cells, their composition changes due to physiological disorders or major diseases such as cardiovascular disease, obesity, Alzheimer's disease or various types of cancer (van Meer, 2005).

Colon cancer tissue was analyzed by MALDI mass spectrometry to interpret abnormal distribution of phospholipids in cancerous regions (Shimma et al., 2007). The HE stained section of colon cancer tissue clearly showed localization of normal, stroma and cancer cells on the tissue sections. When these sections were matrix coated and analyzed by MALDI mass spectrometry, the profile data revealed altered phospholipids distribution between the three aforementioned regions and that one important sphingomyelin (16:0) Na was highly expressed only in cancerous regions.

A number of studies have been conducted to reveal the cel- lular lipid composition of normal and diseased tissue. In a recent paper published by Murphy et al.(2009), the phospholipid compositions of brain and kidney tissues were analyzed by MALDI mass spectrometry. They found that the total ion mass spectra from brain tissue sections differed from the total mass spectra of kidney tissue. In addition, they demonstrated layer specific phospholipid distributions on kidney tissues, with PC $34: 2 \mathrm{~K}$ at $\mathrm{m} / \mathrm{z} 796.6$ being more highly expressed in the cortex and pelvic region of mouse kidneys than the medulla. Conversely, $\mathrm{PC} 40: 6$ at $\mathrm{m} / \mathrm{z} 862.6$ was distributed throughout the region of the medulla and cortex, but was not present in the pelvic region (Murphy et al., 2009).

Related work was conducted by Hayasaka et al. (2008) in which the layer distribution of phospholipids and their contribution to phototransduction were demonstrated in mouse retina by MALDI-QIT-TOF imaging mass spectrometry (Hayasaka, et al., 2008). On frozen retina tissue sections, they found four different PCs with different ion forms and their images demonstrated that PC 34:1 was distributed in the inner nuclear layer and outer plexiform layer, PC 32:0 was present in the outer nuclear layer and inner segment and both PC 38:6 and 40:6 were distributed in the outer segment and pigment epithelium. To confirm their structural information, $\mathrm{ms} / \mathrm{ms}$ analysis was conducted on a tissue section. They compared their MALDI data images with the optical image of the retina section to confirm the layer distribution of phospholipids. Moreover, to overcome the contamination of isotopic peaks of other phospholipids and biomolecules with their lipid of interest, they confirmed each PC image individually and merged images on retina tissue sections.

Similarly, the IMS of phospholipids for different neurological diseases are now receiving increased attention. Approximately $60 \%$ of the dry weight of brain tissue consists of lipids. Any neurological disorder may lead to changes in lipid composition and concentration as they provide stability, fluidity and permeability to the neuronal membrane and are also required for proper function of integral membrane proteins, receptors and ion-channels (Farooqui and Horrocks, 1985; Farooqui et al., 2000; Adibhatla et al., 2006). Research is currently being conducted to identify these changes for the development of precise biomarkers for specific neurological diseases.

Gangliosides are glycerolipids that contain sialic acids and play a major role in neuronal functions. Evidence has shown that gangliosides are directly related to several neurological disorders. For example, GM1 helps in amyloid plaque formation in the brain, which is a major factor of Alzheimer's disease (Ariga et al., 2008). In addition, over expression of GM3 was found to induce neuronal cell death in the immortalized mouse hippocampal cell line HT22 (Sohn et al., 2006), and gangliosides are closely related to neuronal plasticity during neurodevelopment and aging (Mutoh et al., 2006). Chan et al. investigated images of gangliosides in mouse brain tissue sections by MALDI mass spectrometry. They introduced a new ionic liquid matrix that was capable of identifying gangliosides from mouse brain tissue sections without dissociation of sialic acid during the ionization process. Using IMS experiments, they successfully showed the distribution of GM1 at $\mathrm{m} / \mathrm{z} 1546$ and m/z 1574 on the pyramidal neuronal cell layer and the dentate gyrus region, respectively (Chan et al., 2009). These regions play an important role in learning and memory formation (Shors et al., 2001) and are affected during neurodegenerative diseases such as Alzheimer's disease (Jeyaku- 


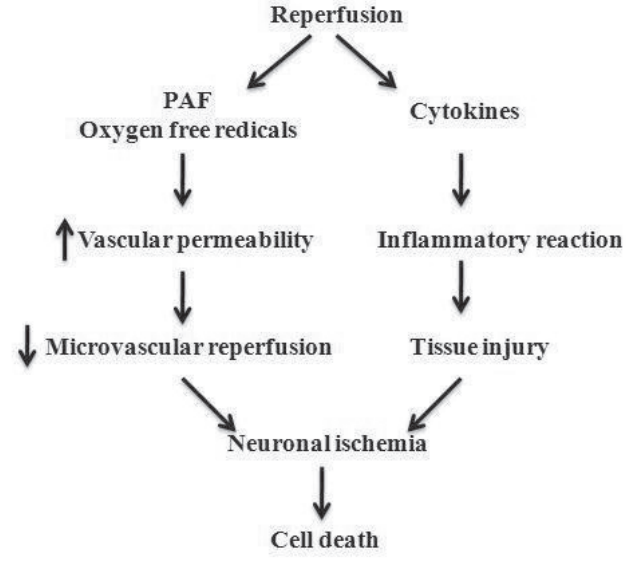

Fig. 5. An overview of neuronal ischemic cell death.

mar et al., 2005).

Cerebral ischemia is a neuronal disease caused by obstruction of blood flow and reduced oxygen that leads to reduced glucose supply to brain cells, resulting in injury and apoptosis. An overview of ischemic cell death is presented in Fig. 5. For better understanding of ischemic injury, mouse models were built and analyzed. Recently, Koizumi et al. (2010) revealed one specific lysophosphatidylcholine (LPC) that was produced due to the activation of $\mathrm{PLA}_{2}$ after ischemic injury. After $\mathrm{HE}$ staining of a focal ischemic injured rat brain tissue section, they compared the phospholipids distribution between injured and normal brain regions by MALDI mass spectrometry. Based on statistical analysis and the images, they showed that LPC (16:0) at $\mathrm{m} / \mathrm{z} 496.3$ was induced after ischemic injury, whereas PC $(34: 1)$ and PC $(34: 1) \mathrm{K}$ were reduced after injury. These findings may be explained by the $\mathrm{Ca}^{+}$dependent activation of $\mathrm{PLA}_{2}$ via NMDA receptor stimulation (Lee et al., 2000). It is well known that LPC plays an important role in inflammation due to its promotion of the cytokine effect (Murugesan et al., 2003),induction of apoptosis (Takahashi et al., 2002), reduction of nitric oxide (Deckert et al., 1998) and induction of PLA ${ }_{2}$ activity, as LPC was found to be accumulated in intra and extra cellular spaces during ischemia (Watanabe and Okada, 2003). The findings of Koizumi et al.(2001), which support those of previous studies, revealed a novel method of biomarker discovery by MALDI IMS.

\section{SUMMARY}

Imaging of drugs and its metabolites have opened a new method of understanding candidate drug specificity and their pharmacodynamics. Using IMS as an analyzing tool has made it more convenient to show the localization and distribution of any targeted drugs and their metabolites. Moreover, MALDI-IMS helps us expose the analysis of small endogenous biomolecules such as lipids directly from tissue sections. This technique not only helps in biological studies by enabling analysis of different disease states, but also assists us in determining the activity of specific drug applications on animals under specific physiological conditions.

Here, we focused on the application of MALDI mass spec- trometry, specifically MALDI IMS, as a powerful tool for understanding drug pharmacology and developing new biomarkers for diagnosis. To enable a better understanding, we presented several recent studies; however, there are still many opportunities to obtain information. We hope this review will help researchers appreciate MALDI IMS and facilitate its use in biological and pharmaceutical studies.

\section{ACKNOWLEDGMENTS}

This paper was supported by Konkuk University in 2010 .

\section{REFERENCES}

Adibhatla, R. M., Hatcher, J. F. and Dempsey, R. J. (2006) Lipids and lipidomics in brain injury and diseases. AAPS J. 8, E314-321.

Aravagiri, M., Teper, Y. and Marder, S. R. (1999) Pharmacokinetics and tissue distribution of olanzapine in rats. Biopharm. Drug Dispos. 20, 369-377.

Ariga, T., McDonald, M. P. and Yu, R. K. (2008) Role of ganglioside metabolism in the pathogenesis of Alzheimer's disease--a review. J. Lipid. Res. 49, 1157-1175.

Caprioli, R. M., Farmer, T. B. and Gile, J. (1997) Molecular imaging of biological samples: localization of peptides and proteins using MALDI-TOF MS. Anal. Chem. 69, 4751-4760.

Chan, K., Lanthier, P., Liu, X., Sandhu, J. K., Stanimirovic, D. and Li, J. (2009) MALDI mass spectrometry imaging of gangliosides in mouse brain using ionic liquid matrix. Anal. Chim. Acta. 639, 57-61.

Chaurand, P., Schwartz, S. A., Reyzer, M. L. and Caprioli, R. M. (2005) Imaging mass spectrometry: principles and potentials. Toxicol. Pathol. 33, 92-101.

Chaurand, P., Stoeckli, M. and Caprioli, R. M. (1999) Direct profiling of proteins in biological tissue sections by MALDI mass spectrometry. Anal. Chem. 71, 5263-5270.

Cornett, D. S., Frappier, S. L. and Caprioli, R. M. (2008) MALDI-FTICR imaging mass spectrometry of drugs and metabolites in tissue. Anal. Chem. 80, 5648-5653.

Deckert, V., Brunet, A., Lantoine, F., Lizard, G., Millanvoye-van Brussel, E., Monier, S., Lagrost, L., David-Dufilho, M., Gambert, P. and Devynck, M. A. (1998) Inhibition by cholesterol oxides of NO release from human vascular endothelial cells. Arterioscler. Thromb. Vasc. Biol. 18, 1054-1060.

Farooqui, A. A. and Horrocks, L. A. (1985) On the role of sulfolipids in mammalian metabolism. Mol. Cell Biochem. 66, 87-95.

Farooqui, A. A., Horrocks, L. A. and Farooqui, T. (2000) Glycerophospholipids in brain: their metabolism, incorporation into membranes, functions, and involvement in neurological disorders. Chem. Phys. Lipids. 106, 1-29.

Hayasaka, T., Goto-Inoue, N., Sugiura, Y., Zaima, N., Nakanishi, H., Ohishi, K., Nakanishi, S., Naito, T., Taguchi, R. and Setou, M. (2008) Matrix-assisted laser desorption/ionization quadrupole ion trap time-of-flight (MALDI-QIT-TOF)-based imaging mass spectrometry reveals a layered distribution of phospholipid molecular species in the mouse retina. Rapid Commun. Mass. Spectrom. 22, 3415-3426.

Jeyakumar, M., Dwek, R. A., Butters, T. D. and Platt, F. M. (2005) Storage solutions: treating lysosomal disorders of the brain. Nat. Rev. Neurosci. 6, 713-725.

Khatib-Shahidi, S., Andersson, M., Herman, J. L., Gillespie, T. A. and Caprioli, R.M. (2006) Direct molecular analysis of whole-body animal tissue sections by imaging MALDI mass spectrometry. Anal. Chem. 78, 6448-6456.

Kim, Y., Shanta, S. R., Zhou, L. H. and Kim, K. P. (2010) Mass spectrometry based cellular phosphoinositides profiling and phospholipid analysis: a brief review. Exp. Mol. Med. 42, 1-11.

Koizumi, S., Yamamoto, S., Hayasaka, T., Konishi, Y., YamaguchiOkada, M., Goto-Inoue, N., Sugiura, Y., Setou, M. and Namba, 
H. (2010) Imaging mass spectrometry revealed the production of lyso-phosphatidylcholine in the injured ischemic rat brain. Neuroscience. 168, 219-225.

Lee, J. M., Grabb, M. C., Zipfel, G. J. and Choi, D. W. (2000) Brain tissue responses to ischemia. J. Clin. Invest. 106, 723-731.

Lee, J. R., Lee, S. J., Kim, T. W., Kim, J. K., Park, H. S., Kim, D. E. Kim, K. P. and Yeo, W. S. (2009) Chemical approach for specific enrichment and mass analysis of nitrated peptides. Anal. Chem. 81, 6620-6626.

Murphy, R. C., Hankin, J. A. and Barkley, R. M. (2009) Imaging of lipid species by MALDI mass spectrometry. J Lipid Res. 50(Suppl), S317-322.

Murugesan, G., Sandhya Rani, M. R., Gerber, C. E., Mukhopadhyay, C., Ransohoff, R. M., Chisolm, G. M. and Kottke-Marchant, K. (2003) Lysophosphatidylcholine regulates human microvascular endothelial cell expression of chemokines. J. Mol. Cell Cardiol. 35 1375-1384.

Mutoh, T., Hirabayashi, Y., Mihara, T., Ueda, M., Koga, H., Ueda, A., Kokura, T. and Yamamoto, H. (2006) Role of glycosphingolipids and therapeutic perspectives on Alzheimer's disease. CNS Neurol. Disord. Drug Targets. 5, 375-380.

Schwamborn, K. and Caprioli, R. M. (2010) Molecular imaging by mass spectrometry--looking beyond classical histology. Nat. Rev. Cancer 10, 639-646.

Shanta, S. R., Zhou, L. H., Park, Y. S., Kim, Y. H., Kim, Y. and Kim, K. P. (2011) Binary Matrix for MALDI Imaging Mass Spectrometry of Phospholipids in Both Ion Modes. Anal Chem. 83, 1252-1259.

Shimma, S., Sugiura, Y., Hayasaka, T., Hoshikawa, Y., Noda, T. and Setou, M. (2007) MALDI-based imaging mass spectrometry revealed abnormal distribution of phospholipids in colon cancer liver metastasis. J. Chromatogr. B. Analyt. Technol. Biomed. Life Sci. 855, 98-103.

Shors, T. J., Miesegaes, G., Beylin, A., Zhao, M., Rydel, T. and Gould, E. (2001) Neurogenesis in the adult is involved in the formation of trace memories. Nature. 410, 372-376.

Sohn, H., Kim, Y. S., Kim, H. T., Kim, C. H., Cho, E. W., Kang, H. Y., Kim, N. S., Ryu, S. E., Lee, J. H. and Ko, J. H. (2006) Ganglioside GM3 is involved in neuronal cell death. FASEB J. 20, 1248-1250.

Sugiura, Y. and Setou, M. (2010) Imaging mass spectrometry for visualization of drug and endogenous metabolite distribution: toward in situ pharmacometabolomes. J. Neuroimmune. Pharmacol. 5, 31-43.

Takahashi, M., Okazaki, H., Ogata, Y., Takeuchi, K., Ikeda, U. and Shimada, K. (2002) Lysophosphatidylcholine induces apoptosis in human endothelial cells through a p38-mitogen-activated protein kinase-dependent mechanism. Atherosclerosis. 161, 387-394.

van Meer, G. (2005) Cellular lipidomics. EMBO J. 24, 3159-3165.

Walch, A., Rauser, S., Deininger, S. O. and Hofler, H. (2008) MALDI imaging mass spectrometry for direct tissue analysis: a new frontier for molecular histology. Histochem. Cell Biol. 130, 421-434.

Wang, H. Y., Jackson, S. N., McEuen, J. and Woods, A. S. (2005) Localization and analyses of small drug molecules in rat brain tissue sections. Anal. Chem. 77, 6682-6686.

Watanabe, M. and Okada, T. (2003) Lysophosphatidylcholine-induced myocardial damage is inhibited by pretreatment with poloxamer 188 in isolated rat heart. Mol. Cell Biochem. 248, 209-215.

Wiseman, J. M., Ifa, D. R., Zhu, Y., Kissinger, C. B., Manicke, N. E., Kissinger, P. T. and Cooks, R. G. (2008) Desorption electrospray ionization mass spectrometry: Imaging drugs and metabolites in tissues. Proc. Natl. Acad. Sci. USA. 105, 18120-18125. 